\begin{tabular}{|c|l|}
\hline Title & Micro droplets generated on a rising bubble through an oppositely charged oil/water interface \\
\hline Author(s) & Uemura, T.; Ueda, Y.; Iguchi, Manabu \\
\hline Citation & $\begin{array}{l}\text { Journal of Visualization, 15(2), 119-124 } \\
\text { https://doi.org/L0.1007/312650-011-0116-8 }\end{array}$ \\
\hline Issue Date & 2012-05 \\
\hline Doc URL & http://hdl.handle.net/2115/52693 \\
\hline Rights & The original publication is available at www.springerlink.com \\
\hline Type & article (author version) \\
\hline File Information & JoV 15-2_119-124.pdf \\
\hline
\end{tabular}

Instructions for use 


\title{
Micro droplets generated on a rising bubble through an oppositely charged oil/water interface
}

\author{
T. Uemura Y. Ueda M. Iguchi
}

the date of receipt and acceptance should be inserted later

\begin{abstract}
The mass transfer between immiscible two-liquid phases can be greatly enhanced by bubbling gas through a reactor. Numerous micro water droplets breaking out from a ruptured water film around a rising bubble through the oil (upper phase)/water (lower phase) interface were demonstrated in the preceding paper (UEMURA T., UEDA Y. And Iguchi M., Europhys. Lett., 92 (2010) 34004). In this letter, we attempt to oppositely charge the oil and water layers, taking into account the findings of the preliminary study (Uemura T., Ueda Y. ANd IGuchi M., J. Visualization, 13 (2010) $85)$. As a result, this study successfully produces more and finer water droplets than the preceding experiments.
\end{abstract}

Keywords Bubble . Immiscible two-liquid interface · Ripple · Electric field . High-speed photography

\section{Introduction}

The investigations of bubble-bursting at a static water surface can be found in a lot of papers (e.g., Knelman et al. 1954; MacIntyre 1972; Boulton-Stone and Blake 1993; Duchemin et al. 2002). In particular, puncturing of viscous bubbles at an air/liquid surface with a sharp needle produces a folding of the film that entraps a torus of air, and most of the resulting rim disintegrates into droplets (see Bird et al. 2010). When a highly viscous liquid bubble is ruptured, radial ripples grow on the bubble surface due to the hydrodynamic instability (see da Silveira et al. 2000).

During the passage of a rising bubble through an oil (upper phase)/water (lower phase) interface, the bubble attracts a column of the water upwards keeping a film of the water around itself. The film and column then flow back into the water layer, and parts of them disintegrate into droplets (see Uemura et al. 2010a; Reiter and Schwerdtfeger

T. Uemura

Department of Mechanical Engineering, Kansai University, Osaka 564-8680, Japan

E-mail: umra@kansai-u.ac.jp

Y. Ueda · M. Iguchi

Division of Materials Science and Engineering, Hokkaido University, Hokkaido 060-8628, Japan

E-mail: y-ueda@eng.hokudai.ac.jp, gaku@eng.hokudai.ac.jp 


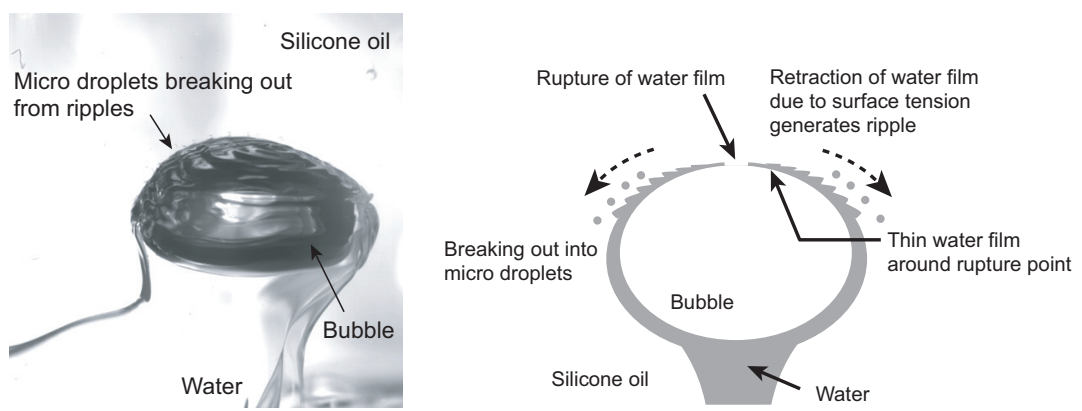

Fig. 1 Numerous micro water droplets break out from concentric ripples generated on a ruptured water film around a rising bubble through the oil/water interface (Reproduced from Uemura et al. 2010a). (Left): Photograph of a rising bubble through the oil/water interface. (Right): Schematic of a breaking out event of droplets from the ripples.

1992a, 1992b). When the water film around the rising bubble ruptures, the water film abruptly shrinks due to the interfacial tension on the air/water interface greater than that on the oil/water interface. The preceding paper of Uemura et al. (2010a) visually demonstrates that this strong retraction forms concentric ripples on the outer interface of the bubble. Due to the propagation of the ripples, numerous micro water droplets were observed to break out from the crest of the fully grown ripples within $2 / 3400 \mathrm{~s}$ because of the relatively weak interface tension on the outer bubble surface (see Fig. 1).

Electric fields induce motion of charged particles or liquids (Ristenpart et al. 2009) and it is utilized in practical fields such as chemical engineering. Likewise, in a steel refining process, interface motion due to electrophoretic forces between immiscible liquids increases interface area so that it would be expected to promote a chemical reaction. In the preceding paper of Uemura et al. (2010b), we observed an oppositely charged oil/water interface by experiment (see also Taylor and McEwan 1965; Melcher and Smith 1969; Taylor 1969). With $33 \mathrm{kV}$ voltage supplied for the silicone oil of $5 \mathrm{cSt}$ viscosity, the attracted interface increases the swell (so-called Taylor cone, see Taylor 1964; de la Mora 2007) which sharpens with time and ends up being torn at the peak point of the elevated interface producing micro droplets.

A key issue for promoting chemical reaction is to produce large numbers of micro droplets from an immiscible two-liquid interface. To do so, we attempt to investigate behaviour of a water film around a rising bubble through an oppositely charged oil/water interface. This target system utilizes the findings of Uemura et al. (2010b) along the lines of the preceding system (see Uemura et al. 2010a).

\section{Experimental setup and procedure}

An instantaneous phenomenon occurring on a bubble surface during the passage of the rising bubble through an immiscible oil/water interface is demonstrated experimentally (see Fig. 2), by using a transparent acrylic cylindrical container (176 mm in inner diameter, $450 \mathrm{~mm}$ in height) with the bottom half $\left(h_{w}=100 \mathrm{~mm}\right.$ from the nozzle exit) filled with deionised (DI) water $\left(\mu_{w}=0.000855 \mathrm{~Pa} \cdot \mathrm{s}\right)$ and the top half $\left(h_{o}=100 \mathrm{~mm}\right)$ with an immiscible silicone oil (viscosity 1 or $5 \mathrm{cSt}$ ). The cylindrical container is surrounded by a larger acrylic rectangular tank filled with tap water so that distortion of 


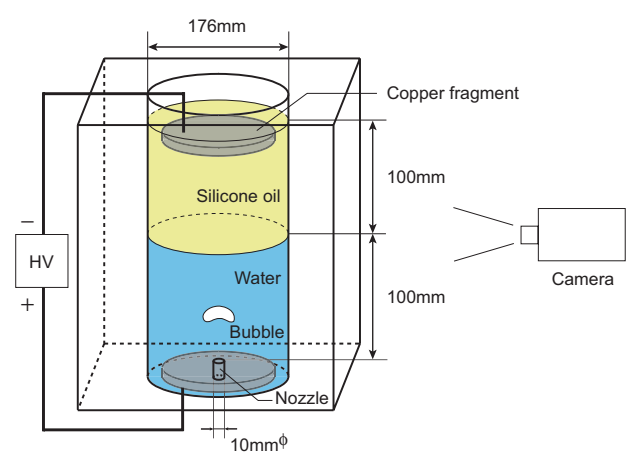

Fig. 2 Schematic diagram of experimental setup. The viscosity of the silicone oil is either 1 or $5 \mathrm{cSt}$.

images due to light refraction can be reduced in a photograph. Copper fragments are inserted into each liquid (at top and bottom) to serve as electrodes where the water layer is positively charged (see Fig. 2). A high DC voltage power supply provides a potential difference between about 0 and $35 \mathrm{kV}$. The inlet bubble is injected into the cylindrical container through a single-hole nozzle, made of brass, with an inner diameter of $d_{N}=10 \mathrm{~mm}$ and a height of $h_{N}=90 \mathrm{~mm}$ longer than the developing distance of the airflow. The inlet flow rate of the bubble is controlled by an air compressor (BEBICOM, Hitachi Co. Ltd.) and measured by using a flowmeter (KOFLOC RK1250). The flow rate of the bubble injection $Q_{g}$ is varied between 5 and $70 \mathrm{~mL} / \mathrm{min}$. A microscale phenomenon instantaneously occurring on the bubble surface during the passage is recorded with a high-speed camera (Phantom Ver. 7.1) whose frame rate is 7,200 frames/s with the shutter speed $75 \mu$ s for the resolution $640 \times 480$ pixels.

\section{Results and discussion}

Figures 3 and 4 show a comparison of micro water droplets formation from the ripples of a ruptured water film around a rising bubble through an oppositely charged oil/water interface between 0 and $20 \mathrm{kV}$ voltage. The viscosity of the silicone oil is $1 \mathrm{cSt}$. In the absence of the voltage, micro water droplets form from the ripples which relate to the Rayleigh-Taylor instability, although the detailed mechanism remains unclear. As shown in Fig. 5, left (reproduced from Uemura et al. 2010a), the concentric ripples propagate and grow on the surface of the bubble and the micro droplets break out from the crest of the fully grown ripples. By zooming-in on the ripple and capturing the image in the white rectangle of Fig. 4 (v), the formation of micro droplets from ripples under $20 \mathrm{kV}$ voltage can be explained. Under the voltage supplied, the electric field forces pinch off the micro droplets from the ripples (see Fig. 5, right) and attracts the droplets toward the minus electrode (see Fig. 6 and Supplementary Movie 1) which is unlike the droplets behaviour under the zero voltage (see Fig. 3 (vi) and Supplementary Movie 2). A greater number and finer water droplets are observed under $20 \mathrm{kV}$ voltage than in the absence of the voltage, as shown in Fig. 7 (droplet atomization occurs in silicone oil $5 \mathrm{cSt}$ viscosity under $25 \mathrm{kV}$ voltage). Note that the water droplets seem to be stretched upwards in Fig. 5, right whereas they are spherical in the absence of 


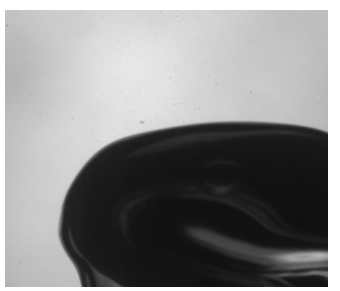

(i) $0 / 7200 \mathrm{~s}$

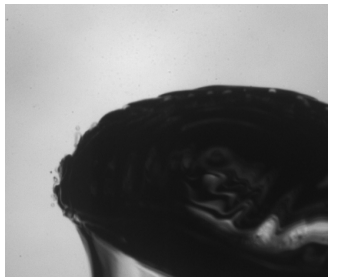

(iv) $20 / 7200 \mathrm{~s}$

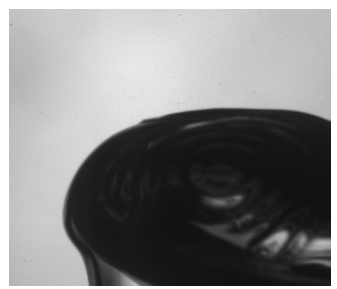

(ii) $8 / 7200 \mathrm{~s}$

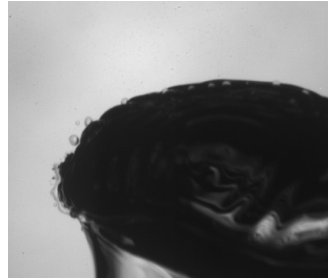

(v) $22 / 7200 \mathrm{~s}$

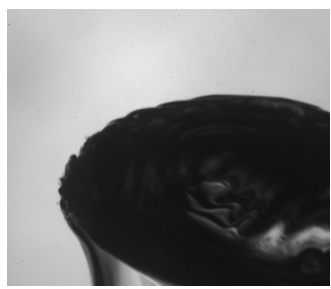

(iii) $17 / 7200 \mathrm{~s}$

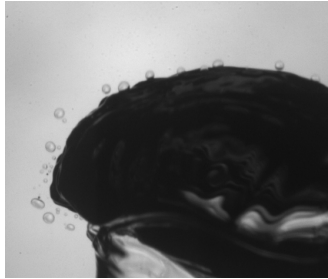

(vi) $32 / 7200 \mathrm{~s}$

Fig. 3 Selected snapshots of micro water droplets breaking out from a ruptured water film around a rising bubble through an oil (1 cSt viscosity)/water interface in the absence of the voltage.

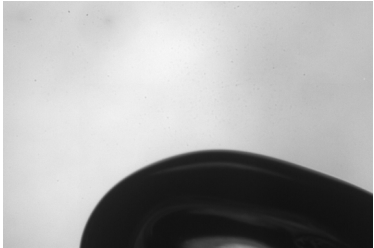

(i) $0 / 7200 \mathrm{~s}$

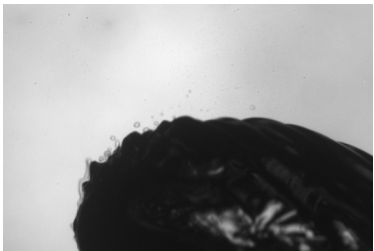

(iv) $34 / 7200 \mathrm{~s}$

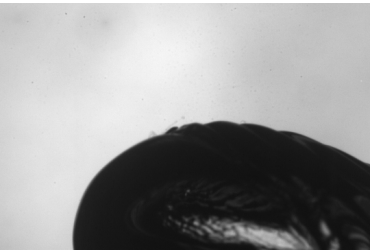

(ii) $26 / 7200 \mathrm{~s}$

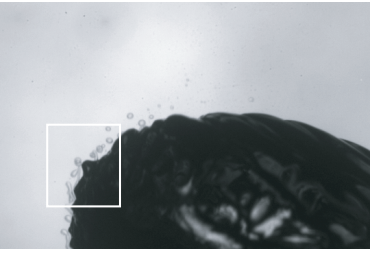

(v) $37 / 7200 \mathrm{~s}$

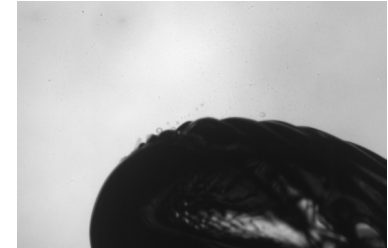

(iii) $29 / 7200 \mathrm{~s}$

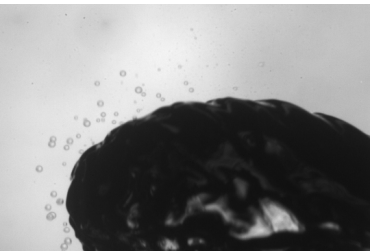

(vi) $56 / 7200 \mathrm{~s}$

Fig. 4 Selected snapshots of micro water droplets breaking out from a ruptured water film around a rising bubble through an oil (1 cSt viscosity)/water interface under $20 \mathrm{kV}$ voltage. The white rectangle in (v) is magnified in Fig. 5 , right.

the voltage (Fig. 5, left). This means that the apex of the water droplet is attracted upwards due to the electric field.

To explore possible diameter of generated micro water droplets, we systematically estimate the electric force $F_{E}=q E$ in which the magnitude of charge on the droplet $q$ depends on the details of charge transfer between the droplet and the interface contacted (i.e., copper electrode) (see Ristenpart et al. 2009). Following Ristenpart et al. (2009), we determine $q$ using Stokes' drag $F_{D}$ for a sphere and the measured 

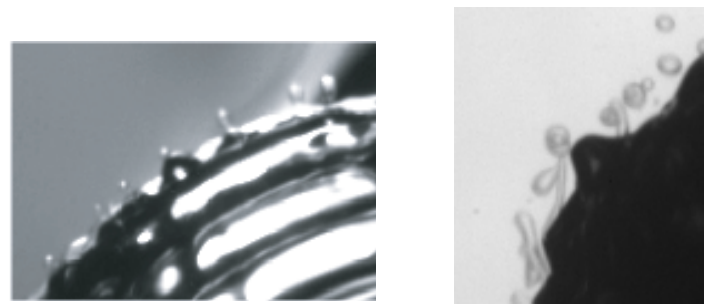

Fig. 5 Formation of micro water droplets from ripples. (Left): Top view of the bubble surface in the absence of voltage. Reproduced from Uemura et al. (2010a). (Right): Magnified view in the white rectangle of Fig. 4 (v). Micro droplets forcibly break out from the ripple due to an electric field supplied under $20 \mathrm{kV}$ voltage.

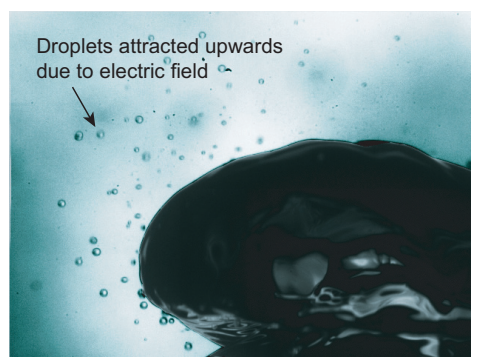

Fig. 6 Electric field attracts micro water droplets upward (silicone oil is $1 \mathrm{cSt}$ viscosity). Velocities of the droplets are measured in a series of snapshots.

velocities of droplets which are attracted upwards due to $F_{E}$ (see Fig. 6), i.e.,

$$
q=(\pi / 6) d_{p}^{3} g\left(\rho_{w}-\rho_{o}\right)+F_{D} / E
$$

with $C_{D}=(8 / \pi)\left[F_{D} /\left(\rho_{o} d_{p}{ }^{2} U_{p}^{2}\right)\right]$, where $d_{p}$ is the diameter of the droplet, $\rho_{w}$ and $\rho_{o}$ are the densities of the water and the silicone oil, respectively. The electric field $E$ at the droplet position is determined by the distance from the minus electrode (upper oil surface), i.e., $E \approx V / h_{o}$. Under low Reynolds number, the drag coefficient $C_{D}$ is represented as $C_{D}=24 / R e_{p}$ with $R e_{p}=U_{p} d_{p} / \nu_{o}$. The movement of an attracted micro water droplet was measured, where the droplet with $d_{p}=0.23 \mathrm{~mm}$ constantly moved upward with the velocity of $451 \mathrm{~mm} / \mathrm{s}$. Then, Eq.(1) estimates the magnitude of charge on the droplet as $q=4.0 \times 10^{-12} \mathrm{C}$.

The force balance on the micro water droplet breaking out from the water film around the rising bubble through the oppositely charged oil/water interface is sketched in Fig. 8. Here, the spherical micro droplet having diameter $d_{p}$ is assumed to break out at the position on the bubble surface where the radius of curvature is $R$ (The assumption about the spherical shape of the droplet would be valid because $d_{p}$ is at most $0.4 \mathrm{~mm}$ as seen in Fig. 7 ). The propagating ripple with the velocity $U_{R}$ pinches off the droplet due to the centrifugal force $F_{C} \approx(\pi / 6) \rho_{w} d_{p}^{3} U_{R}^{2} / R$ (the distance of the mass centre of the droplet from the water film is vanishingly smaller than $R$ ) and the electric force $F_{E}=q E$ against the gravitational force $F_{G}=(\pi / 6) d_{p}^{3} g\left(\rho_{w}-\rho_{o}\right)$ including the buoyancy force. In the absence of the voltage, the droplet breaks out from the water film when $F_{C}$ becomes larger than the sum of $F_{G}$ and the capillary force $F_{\sigma}$, i.e., the droplet 

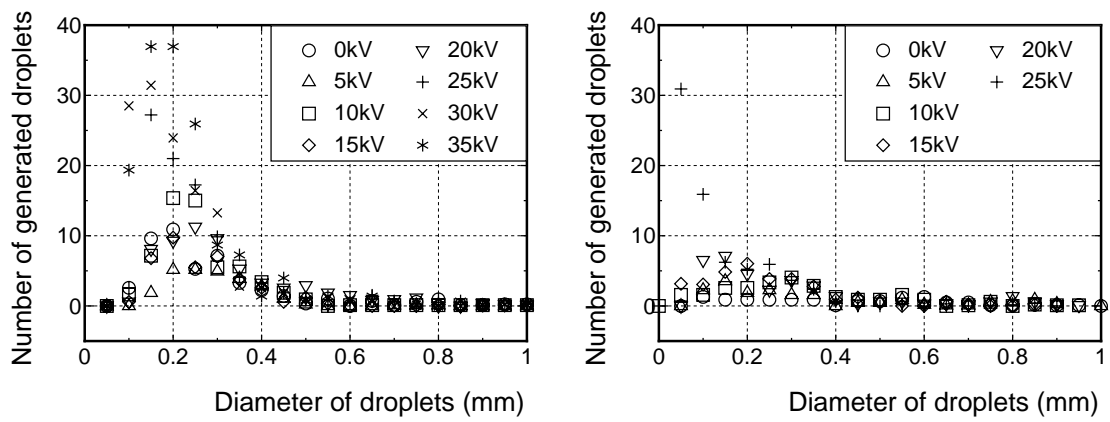

Fig. 7 Distributions of averaged diameter of generated micro droplets from the ripple under various voltages for silicone oils of (left): $1 \mathrm{cSt}$ or (right): $5 \mathrm{cSt}$ viscosity.

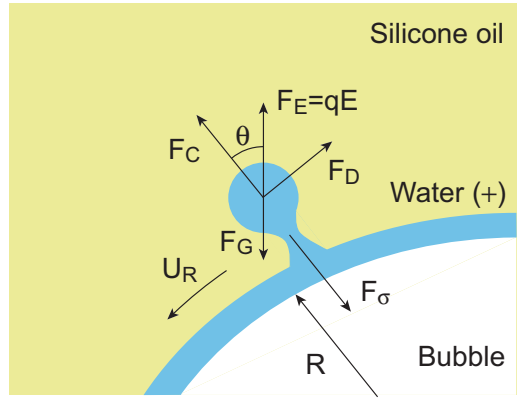

Fig. 8 Sketch of a micro water droplet breaking from a water film around a rising bubble through an oil/water interface.

size under the zero voltage, $d_{p 0}$, is satisfied with $(\pi / 6) \rho_{w}\left(U_{R 0}^{2} / R\right) d_{p 0}^{3}-(\pi / 6) d_{p 0}^{3} g\left(\rho_{w}-\right.$ $\left.\rho_{o}\right) \cos \theta=F_{\sigma}$, where $U_{R 0}$ denotes the propagation velocity of a ripple in the absence of the electric field. Under the voltage supplied, the electric field $\left(E \approx V / h_{o}\right)$ affects the force balance as $(\pi / 6) \rho_{w}\left(U_{R}^{2} / R\right) d_{p}^{3}-(\pi / 6) d_{p}^{3} g\left(\rho_{w}-\rho_{o}\right) \cos \theta+q E \cos \theta=F_{\sigma}$. An assumption that the capillary force $F_{\sigma}$ would be independent of the electric field estimates the droplet size $d_{p}$ under a voltage $V$ as

$$
d_{p}^{3}=\frac{1}{\psi} \quad \psi_{0} d_{p 0}^{3}-\frac{6}{\pi} \frac{V}{h_{o}} q \cos \theta
$$

in which $\psi_{0}=\rho_{w}\left(U_{R 0}^{2} / R\right)-\left(\rho_{w}-\rho_{o}\right) g \cos \theta_{0}$ and $\psi=\rho_{w}\left(U_{R}^{2} / R\right)-\left(\rho_{w}-\rho_{o}\right) g \cos \theta$. As shown in Fig. 8, the tangential component of the electric force, $F_{E} \sin \theta$, decreases the propagation velocity of the ripple so that $U_{R}$ under the electric field is smaller than $U_{R 0}$ in the absence of it. Indeed, the values of $U_{R}$ measured by extracting from the images are between 1464 and $2009 \mathrm{~mm} / \mathrm{s}$ for $1 \mathrm{cSt}$ silicone oil under $20 \mathrm{kV}$ voltage (see Fig. 4 and Supplementary Movie 1), whereas $U_{R 0}$ is between around 2000 and $4000 \mathrm{~mm} / \mathrm{s}$ (see Fig. 3 and Supplementary Movie 2). Here, $\theta$ is assumed to be the same as $\theta_{0}$ and it is estimated from Fig. 4a of Uemura et al. (2010a) where micro droplets break out from the ripple around $0^{\circ} \quad \theta_{0} \quad 114^{\circ}$. For the experiment using $1 \mathrm{cSt}$ under $20 \mathrm{kV}$ voltage, we use the mean values as $U_{R}=1737 \mathrm{~mm} / \mathrm{s}, U_{R 0}=3000 \mathrm{~mm} / \mathrm{s}$ and 
$\theta=\theta_{0}=57^{\circ}$ together with $q=4.0 \times 10^{-12} \mathrm{C}$ and $h_{o}=100 \mathrm{~mm}$, and then Eq.(2) yields $d_{p}^{3}=\left(3.0 d_{p 0}^{3}-1.4 \times 10^{-11}\right)$. Now, we look at Fig. 7 (left) and find that $d_{p 0} \approx 0.2 \mathrm{~mm}$ in the absence of the voltage. Then, Eq.(2) gives $d_{p}=0.21 \mathrm{~mm}$ for $20 \mathrm{kV}$ voltage which is found to be reasonable against the results of Fig. 7 (left). Of course, the estimated value of $d_{p}$ is in good agreement with the above-mentioned measured value $d_{p}$ of the attracted droplet upwards. As observed in Figs. 7, more and finer micro water droplets can be successfully produced against the zero voltage experiments when the supplied voltage exceeds a critical value.

\section{Concluding remarks}

In summary, this study visualizes micro droplets breaking out from a water film around a rising bubble through an oppositely charged oil/water interface. The electric field supplied forces to pinch off the micro droplets from the crest of a ripple and attracts the droplets upwards. Equation (2) predicts the droplet size under a certain supplied voltage based upon that in the absence of the voltage. A key remark on this letter is that more and finer micro droplets can be successfully produced against the preceding experiment of Uemura et al. (2010a) in the absence of the voltage when the supplied voltage exceeds a critical value. This phenomenon increases in total surface area of generated micro droplets so that mass transfer would be expected to be enhanced but it needs to be tested. Further theoretical investigation is desired to resolve the detailed mechanism of pinching off the micro water droplets under strong supplied voltage.

\section{References}

1. Bird JC, de Ruiter R, Courbin L, Stone HA (2010) Daughter bubble cascades produced by folding of ruptured thin films. Nature 465: 759-762.

2. Boulton-Stone JM, Blake JR (1993) Gas bubbles bursting at a free surface. J Fluid Mech 254: $437-466$

3. da Silveira R, Chä̈eb S, Mahadevan L (2000) Rippling Instability of a Collapsing Bubble. Science 287: 1468-1471

4. de la Mora JF (2007) The Fluid Dynamics of Taylor Cones. Annu Rev Fluid Mech 39: $217-243$

5. Duchemin L, Popinet S, Josserand C, Zaleski S (2002) Jet formation in bubbles bursting at a free surface. Phys Fluids 14: 3000-3008

6. Knelman F, Dombrowski N, Newitt, DM (1954) Mechanism of the Bursting Bubbles. Nature 173: 261

7. MacIntyre F (1972) Flow Patterns in Breaking Bubbles. J Geophys Res 77: 5211-5228

8. Melcher JR, Smith Jr CV (1969) Electrohydrodynamic Charge Relaxation and Interfacial Perpendicular-field Instability. Phys Fluids 12: 778-790

9. Reiter G, Schwerdtfeger K (1992a) Observations of physical phenomena occurring during passage of bubbles through liquid/liquid interface. ISIJ International 32: 50-56

10. Reiter G, Schwerdtfeger K (1992b) Characteristics of entrainment at liquid/liquid interfaces due to rising bubbles. ISIJ International 32: 57-65

11. Ristenpart WD, Bird JC, Belmonte A, Dollar F, Stone HA (2009) Non-coalescence of oppositely charged drops. Nature 461: 377-380

12. Taylor G (1964) Disintegration of Water Drops in an Electric Field. Proc R Soc Lond A 280: $383-397$

13. Taylor G (1969) Electrically Driven Jets. Proc R Soc Lond A 313: 453-475

14. Taylor GI, McEwan AD (1965) The Stability of a Horizontal Fluid Interface in a Vertical Electric Field. J Fluid Mech 22: 1-15

15. Uemura T, Ueda Y, Iguchi M (2010a) Ripples on a rising bubble through an immiscible two-liquid interface generate numerous micro droplets. Europhys Lett 92: 34004

16. Uemura T, Ueda Y, Iguchi M (2010b) Behavior of an oppositely charged oil-water interface. $\mathrm{J}$ Vis 13: $85-87$ 